\title{
THE USE OF ADJUSTED TRANSFER RATES IN QUEUEING NETWORKS WITH BACKGROUND LOAD
}

\section{Hans Daduna, Universität Hamburg Rolf Schassberger, TU Braunschweig}

Product form networks are well suited to model the behaviour of complex production systems if quick and easy to apply analytical methods of performance analysis are requested for. When studying only a part of the network it is common to isolate this part and modeling the load, which is put on the subnetwork from external modes by independent Poisson processes. It has been observed in some special models of telecommunication theory that these "background load processes" may be substituted by carefully adjusting the service rate ("transfer rate") of the nodes.

This could be done in a way that the first-order quantities as throughput, mean transfer time, mean waiting time, etc., of the first system are truly reproduced by the system with "adjusted transfer rates". We first generalize this "method of adjusted transfer rates" and then show that the assumptions which are put on the system to reproduce exactly first-order performance characteristics are sufficient for reproducing transfer time characteristics of higher order, even transfer time quantiles - as long as these are accessable by analytical methods in considering overtake-free paths. We present examples which show that such paths appear naturally e.g. in modeling job-shop like queueing systems. 